quatrième série-tome 42 fascicule 2 mars-avril 2009

$$
\begin{aligned}
& \text { ANNALES } \\
& \text { SCIENTIFIQUES } \\
& \text { de } \\
& \text { L'ECOLE } \\
& \text { NORMALE } \\
& \text { SUPÉRIEURE }
\end{aligned}
$$

Eike LAU

A duality theorem for Dieudonné displays 
Ann. Scient. Éc. Norm. Sup.

$4^{\text {e }}$ série, t. 42,2009 , p. 241 à 259

\title{
A DUALITY THEOREM FOR DIEUDONNÉ DISPLAYS
}

\author{
BY EIKE LAU
}

Abstract. - We show that the Zink equivalence between $p$-divisible groups and Dieudonné displays over a complete local ring with perfect residue field of characteristic $p$ is compatible with duality. The proof relies on a new explicit formula for the $p$-divisible group associated to a Dieudonné display.

RÉSUMÉ. - Nous montrons que l'équivalence de Zink entre les groupes $p$-divisibles et les displays de Dieudonné sur un anneau local complet à corps résiduel parfait de caractéristique $p$ est compatible avec la dualité. La preuve repose sur une nouvelle formule explicite pour le groupe $p$-divisible associé à un display de Dieudonné.

\section{Introduction}

Let $R$ be a complete local ring with maximal ideal $\mathfrak{m}$ and perfect residue field $k$ of positive characteristic $p$. If $p=2$ we assume that $p R=0$.

As a generalisation of classical Dieudonné theory, Th. Zink defines in [11] a category of Dieudonné displays over $R$ and shows that it is equivalent to the category of $p$-divisible groups over $R$. In the present article we give a unified formula for the group associated to a Dieudonné display and apply it to show that the equivalence is compatible with the natural duality operations on both sides. This is not clear from the original construction because that depends on decomposing a $p$-divisible group into its étale and infinitesimal part, which is not preserved under duality.

Let us recall the definition of a Dieudonné display. There is a unique subring $\mathbb{W}(R)$ of the Witt ring $W(R)$ that is stable under its Frobenius $f$ and Verschiebung $v$, that surjects onto $W(k)$, and that contains an element $x \in W(\mathfrak{m})$ if and only if the components of $x$ converge to zero $\mathfrak{m}$-adically. In [11] the ring $\mathbb{W}(R)$ is denoted by $\widehat{W}(R)$. Let $\mathbb{I}_{R}$ be the kernel of the natural homomorphism $\mathbb{W}(R) \rightarrow R$. A Dieudonné display over $R$ is a quadruple

$$
\mathscr{P}=\left(P, Q, F, F_{1}\right)
$$

where $P$ is a finite free $\mathbb{W}(R)$-module, $Q$ a submodule containing $\mathbb{I}_{R} P$ such that $P / Q$ is a free $R$-module, $F: P \rightarrow P$ and $F_{1}: Q \rightarrow P$ are $f$-linear maps such that $F_{1}(v(w) x)=w F(x)$ 
for $x \in P$ and $w \in \mathbb{W}(R)$, and the image of $F_{1}$ generates $P$. Dieudonné displays over $k$ are equivalent to Dieudonné modules $(P, F, V)$ where $Q=V(P)$ and $F_{1}=V^{-1}$.

Our formula is based on viewing both $p$-divisible groups and the modules $P, Q$ as abelian sheaves for the flat topology on the opposite category of all $R$-algebras $S$ with the following properties: the nilradical $\mathcal{N}(S)$ is a nilpotent ideal, it contains $\mathfrak{m} S$, and $S / \mathcal{N}(S)$ is a union of finite dimensional $k$-algebras; see section 1 for details. With that convention, the equivalence functor BT from Dieudonné displays to $p$-divisible groups is given by

$$
\operatorname{BT}(\mathscr{P})=\left[Q \stackrel{F_{1} \text {-incl }}{\longrightarrow} P\right] \stackrel{L}{\otimes} \mathbb{Q}_{p} / \mathbb{Z}_{p}
$$

where $[Q \rightarrow P]$ is a complex of sheaves in degrees 0,1 . In other words, the cohomology of the right hand side of $(\star)$ vanishes outside degree zero and the zero-th cohomology is the $p$-divisible group associated to $\mathscr{P}$. Instead of the flat topology one could also use the indétale topology, but for some arguments the former is more convenient.

Before stating the main result let us recall the duality of Dieudonné displays. We have the special Dieudonné display $\mathscr{G}_{m}=\left(\mathbb{W}(R), \mathbb{I}_{R}, f, v^{-1}\right)$ that corresponds to the formal multiplicative group $\widehat{\mathbb{G}}_{m}$. A bilinear form $\mathscr{P}^{\prime} \times \mathscr{P} \rightarrow \mathscr{G}_{m}$ is a bilinear map $\alpha: P^{\prime} \times P \rightarrow \mathbb{W}(R)$ satisfying $\alpha\left(x^{\prime}, x\right)=v\left(\alpha\left(F_{1}^{\prime} x^{\prime}, F_{1} x\right)\right)$ for $x^{\prime} \in Q^{\prime}$ and $x \in Q$. For every $\mathscr{P}$ there is a dual $\mathscr{P}^{t}$ equipped with a perfect bilinear form $\mathscr{P}^{t} \times \mathscr{P} \rightarrow \mathscr{G}_{m}$, which determines $\mathscr{P}^{t}$ uniquely. The Serre dual of a $p$-divisible group $G$ is denoted by $G^{\vee}$.

\section{Theorem. - For every Dieudonné display $\mathscr{P}$ over $R$ there is a natural isomorphism}

$$
\Psi: \operatorname{BT}\left(\mathscr{P}^{t}\right) \cong \mathrm{BT}(\mathscr{P})^{\vee} .
$$

The proof is independent of the fact that the functor BT from Dieudonné displays to $p$-divisible groups defined by $(\star)$ is actually an equivalence. Let us indicate how to define the homomorphism $\Psi$. Denote by $Z(\mathscr{P})$ the complex $[Q \rightarrow P]$ in $(\star)$. To the tautological bilinear form $\mathscr{P}^{t} \times \mathscr{P} \rightarrow \mathscr{G}_{m}$ one can directly assign a homomorphism of complexes $Z\left(\mathscr{P}^{t}\right) \otimes Z(\mathscr{P}) \rightarrow Z\left(\mathscr{G}_{m}\right)$, which gives after tensoring twice with $\mathbb{Q}_{p} / \mathbb{Z}_{p}$ a homomorphism

$$
\mathrm{BT}\left(\mathscr{P}^{t}\right) \stackrel{L}{\otimes} \mathrm{BT}(\mathscr{P}) \rightarrow \mathrm{BT}\left(\mathscr{G}_{m}\right) \stackrel{L}{\otimes} \mathbb{Q}_{p} / \mathbb{Z}_{p} \cong \widehat{\mathbb{G}}_{m}[1] .
$$

By the cohomological theory of biextensions, such a homomorphism is equivalent to a homomorphism $\Psi$ as above. That $\Psi$ is an isomorphism must be shown only if the group $\operatorname{BT}(\mathscr{P})$ is étale or of multiplicative type or bi-infinitesimal. The first two cases are straightforward; the bi-infinitesimal case relies on the theorem of Cartier [3] on the Cartier dual of the Witt ring functor.

Over arbitrary rings in which $p$ is nilpotent, infinitesimal $p$-divisible groups are equivalent to displays according to [13] and [5]. The bi-infinitesimal case of the above theorem is closely related to the duality theorem in [13] for the display associated to a bi-infinitesimal $p$-divisible group. This in turn has been anticipated by Norman [10] who shows a similar duality theorem for the Cartier module of a bi-infinitesimal $p$-divisible group, provided the module is displayed (which is always the case by the said equivalence). These duality results all depend on the theory of biextensions developed in [9], that appears here in the cohomological form it was given in SGA 7. 
The present proof that the functor BT defined by $(\star)$ is an equivalence of categories consists in verifying that it reproduces the equivalence constructed in [11]. However, it should be possible to relate the crystals associated to a Dieudonné display $\mathscr{P}$ and to the $p$-divisible group $\mathrm{BT}(\mathscr{P})$. Then the fact that $\mathrm{BT}$ is an equivalence will follow directly from the Grothendieck-Messing deformation theory of $p$-divisible groups [8], and the duality theorem for Dieudonné displays will be related to the crystalline duality theorem [1]. We hope to return to this point soon. Let us also note that Caruso [4] proved a duality theorem for Breuil modules of finite flat $p$-group schemes [2] by using the crystalline duality theorem. Breuil modules of $p$-divisible groups are related to Dieudonné displays by [12].

This article is organised as follows. In Section 1 the formula for BT is explained, in Section 2 it is shown to give an equivalence of categories, in Section 3 the duality theorem is proved, and Section 4 is concerned with functoriality in the base. In an appendix we discuss briefly the deformational duality theorem [7] since variants of it are used in the text.

\section{Acknowledgement}

The author is grateful to W. Messing and Th. Zink for many valuable discussions and to one of the referees for pointing out that Section 4 was missing.

\section{Exposition of the main formula}

We begin with a number of general definitions and notations. Let $p$ be a prime. For any ring $A$ let $W(A)$ be the ring of $p$-Witt vectors and $I_{A}$ the kernel of the first Witt polynomial $w_{0}: W(A) \rightarrow A$. If $A$ is perfect of characteristic $p, I_{A}$ is generated by $p$. Let $f$ be the Frobenius of $W(A)$ and $v$ the Verschiebung. If $\mathfrak{a} \subset A$ is a nilpotent ideal, let $\widehat{W}(\mathfrak{a}) \subseteq W(\mathfrak{a})$ be the subgroup of Witt vectors with only finitely many non-zero components. More generally, if $A$ is $\mathfrak{a}$-adically complete and separated, let $\widehat{W}(\mathfrak{a}) \subseteq W(\mathfrak{a})$ be the subgroup of Witt vectors whose components converge to zero $\mathfrak{a}$-adically; in other words, $\widehat{W}(\mathfrak{a})=\lim \widehat{W}\left(\mathfrak{a} / \mathfrak{a}^{n}\right)$. In any case $\widehat{W}(\mathfrak{a})$ is an ideal in $W(A)$.

Definition 1.1. - Let $A$ be a ring and $\mathfrak{a} \subset A$ an ideal. The pair $(A, \mathfrak{a})$ is called admissible if $A$ is $\mathfrak{a}$-adically complete and separated and $A / \mathfrak{a}$ is perfect of characteristic $p$. If $p=2$ we also require that $p A=0$.

Lemma 1.2. - If $(A, \mathfrak{a})$ is admissible then there is a unique $f$-stable subring $\mathbb{W}(A)$ of $W(A)$ such that $\mathbb{W}(A) \cap W(\mathfrak{a})=\widehat{W}(\mathfrak{a})$ and $\mathbb{W}(A)$ maps surjectively onto $W(A / \mathfrak{a})$. The subring $\mathbb{W}(A)$ is also stable under $v$.

This is proved in [11] if $A$ is noetherian and $A / \mathfrak{a}$ is a field, but neither of these assumptions is used in the proof. $\mathbb{W}(A)$ is constructed as follows: Since $A / \mathfrak{a}$ is perfect, the projection $W(A) \rightarrow W(A / \mathfrak{a})$ has a unique splitting, necessarily $f$-equivariant, thus an $f$-equivariant decomposition of abelian groups $W(A) \cong W(A / \mathfrak{a}) \oplus W(\mathfrak{a})$, under which $\mathbb{W}(A)$ is mapped to $W(A / \mathfrak{a}) \oplus \widehat{W}(\mathfrak{a})$. The condition that 2 is invertible or zero in $A$ is only needed to guarantee that $\mathbb{W}(A)$ is $v$-stable. We have

$$
\mathbb{W}(A)=\lim _{\longleftarrow} \mathbb{W}\left(A / \mathfrak{a}^{n}\right)
$$

by uniqueness or by the construction. Let $\mathbb{I}_{A}$ be the kernel of $w_{0}: \mathbb{W}(A) \rightarrow A$. 
Definition 1.3. - Assume that $(A, \mathfrak{a})$ is admissible. A Dieudonné display over $A$ is a quadruple $\mathscr{P}=\left(P, Q, F, F_{1}\right)$ such that

$P$ is a finitely generated projective $\mathbb{W}(A)$-module,

$Q$ is a submodule of $P$ containing $\mathbb{I}_{A} P$,

$P / Q$ is projective as an $A$-module,

$F: P \rightarrow P$ and $F_{1}: Q \rightarrow P$ are $f$-linear maps,

$F_{1}(v(w) x)=w F(x)$ for $w \in \mathbb{W}(A)$ and $x \in P$,

$F_{1}(Q)$ generates $P$ as a $\mathbb{W}(A)$-module.

These axioms also imply $F(x)=p F_{1}(x)$ for $x \in Q$.

Remarks. - (1) Every pair of $\mathbb{W}(A)$-modules $(P, Q)$ satisfying the first three of the above conditions admits a decomposition $P=L \oplus T$ such that $Q=L \oplus \mathbb{I}_{A} T$, called normal decomposition. Its existence is straightforward if $\mathfrak{a}=0$, thus $A$ perfect; if $\mathfrak{a}$ is nilpotent one can use that $\widehat{W}(\mathfrak{a})$ is nilpotent as well; the general case follows by passing to the limit.

(2) For a $\mathbb{W}(A)$-module $M$ let $M^{(1)}=\mathbb{W}(A) \otimes_{f, \mathbb{W}(A)} M$, and for an $f$-linear homomorphism of $\mathbb{W}(A)$-modules $F: M \rightarrow N$ let $F^{\sharp}: M^{(1)} \rightarrow N$ be its linearisation. In analogy with [13] Lemma 9, the structure of a Dieudonné display on a pair $(P, Q)$ as above with given normal decomposition $P=L \oplus T$ is equivalent to the isomorphism

$$
\left(F_{1}^{\sharp}, F^{\sharp}\right): L^{(1)} \oplus T^{(1)} \stackrel{\sim}{\longrightarrow} P .
$$

(3) We have the following notion of base change. If $(A, \mathfrak{a})$ and $(B, \mathfrak{b})$ are admissible pairs, every ring homomorphism $g: A \rightarrow B$ with $g(\mathfrak{a}) \subseteq \mathfrak{b}$ induces a ring homomorphism $\mathbb{W}(g)$ : $\mathbb{W}(A) \rightarrow \mathbb{W}(B)$. The base change of a Dieudonné display $\mathscr{P}$ over $A$ by $g$ is then $\mathscr{P}_{B}=$ $\left(P_{B}, Q_{B}, F_{B}, F_{1, B}\right)$ where

$$
P_{B}=\mathbb{W}(B) \otimes_{\mathbb{W}(A)} P, \quad Q_{B}=\operatorname{Ker}\left(P_{B} \rightarrow B \otimes_{A} P / Q\right),
$$

and $F_{B}, F_{1, B}$ are the unique $f$-linear extensions of $F, F_{1}$, whose existence follows from a normal decomposition as explained in [13] Definition 20.

(4) For a Dieudonné display $\mathscr{P}$ over $A$ there is a unique $\mathbb{W}(A)$-linear map $V^{\sharp}: P \rightarrow P^{(1)}$ such that $V^{\sharp}\left(F_{1} x\right)=1 \otimes x$ for $x \in Q$, cf. [13] Lemma 10. Uniqueness is clear; if $P=L \oplus T$ is a normal decomposition, $V^{\sharp}$ can be defined to be

$$
P \stackrel{\left(F_{1}^{\sharp}, F^{\sharp}\right)^{-1}}{\longrightarrow} L^{(1)} \oplus T^{(1)} \stackrel{(1, p)}{\longrightarrow} L^{(1)} \oplus T^{(1)}=P^{(1)} .
$$

We have $F^{\sharp} V^{\sharp}=p$ and $V^{\sharp} F^{\sharp}=p$. If $A$ is perfect, $F_{1}$ is bijective, and its inverse defines an $f^{-1}$-linear map $V: P \rightarrow P$ whose linearisation is $V^{\sharp}$.

Assume now that $R$ is a local ring with maximal ideal $\mathfrak{m}$ and residue field $k$ such that $(R, \mathfrak{m})$ is admissible, i.e. $R$ is $\mathfrak{m}$-adically complete, $k$ is perfect of characteristic $p$, and $p=2$ implies $p R=0$.

Definition 1.4. - Let $\mathcal{C}_{R}$ be the category of all $R$-algebras $S$ such that the nilradical $\mathcal{N}(S)$ is nilpotent, $\mathcal{N}(S)$ contains $\mathfrak{m} S$, and $S_{\text {red }}=S / \mathcal{N}(S)$ is a union of finite dimensional, necessarily étale, $k$-algebras. 
The last condition implies that $S_{\text {red }}$ is perfect, hence $(S, \mathcal{N}(S))$ is admissible, and $\mathbb{W}(S)$ is defined. The following stability properties of $\mathcal{C}_{R}$ are easily established: If $S^{\prime} \leftarrow S \rightarrow S^{\prime \prime}$ are homomorphisms in $\mathcal{C}_{R}$, then $S^{\prime} \otimes_{S} S^{\prime \prime}$ lies in $\mathcal{C}_{R}$; if $S \in \mathcal{C}_{R}$ and $S \rightarrow S^{\prime}$ is a finite ring homomorphism, then $S^{\prime} \in \mathcal{C}_{R}$; if $S \in \mathcal{C}_{R}$ and $S \rightarrow S_{1} \rightarrow S_{2} \rightarrow \ldots$ is an infinite sequence of étale ring homomorphisms, then $\underset{\lim }{\longrightarrow} S_{i}$ lies in $\mathcal{C}_{R}$.

Let $\widetilde{\mathcal{C}}_{R}$ be the category of abelian sheaves on $\mathcal{C}_{R}^{\text {op }}$ for the fpqc topology, i.e. coverings of $S \in \mathcal{C}_{R}$ are finite families of homomorphisms $S \rightarrow S_{i}$ in $\mathcal{C}_{R}$ such that $S \rightarrow \prod S_{i}$ is faithfully flat. The category of $p$-divisible groups over $R$ is naturally a full exact subcategory of $\widetilde{\mathcal{C}}_{R}$ that is stable under extensions. If $\mathscr{P}$ is a Dieudonné display over $R$, base change of Dieudonné displays makes $P$ and $Q$ into abelian presheaves on $\mathcal{C}_{R}^{\text {op }}$, i.e. for $S \in \mathcal{C}_{R}$ we put $P(S)=$ $P_{S}$ and $Q(S)=Q_{S}$. Note that the presheaf $Q$ is determined by the modules $Q \subseteq P$ but not by the module $Q$ alone. The homomorphisms $F$ and $F_{1}$ induce homomorphisms of the associated presheaves which we denote by the same letters. By the following lemma, $P$ and $Q$ are in fact sheaves.

Lemma 1.5. - For a faithfully flat homomorphism $S \rightarrow T$ in $\mathcal{C}_{R}$ the natural sequence $0 \rightarrow \mathbb{W}(S) \rightarrow \mathbb{W}(T) \rightrightarrows \mathbb{W}\left(T \otimes_{S} T\right)$ is exact.

Proof. - The analogous assertion with $W$ in place of $\mathbb{W}$ is clear, cf. [13] Lemma 30. One easily checks that $\mathbb{W}(S)=\mathbb{W}(T) \cap W(S)$, and the lemma follows.

Definition 1.6. - If $\mathscr{P}$ is a Dieudonné display over $R$, let

$$
Z(\mathscr{P})=\left[Q \stackrel{F_{1} \text {-incl }}{\longrightarrow} P\right]
$$

as a complex in $\widetilde{\mathcal{C}}_{R}$ in degrees 0,1 and

$$
\mathrm{BT}(\mathscr{P})=Z(\mathscr{P}) \otimes^{L} \mathbb{Q}_{p} / \mathbb{Z}_{p}
$$

in the derived category $D\left(\widetilde{\mathcal{C}}_{R}\right)$.

Explicitly $\mathrm{BT}(\mathscr{P})$ can be represented by the tensor product $Z(\mathscr{P}) \otimes\left[\mathbb{Z} \rightarrow \mathbb{Z}\left[\frac{1}{p}\right]\right]$ sitting in degrees $-1,0,1$.

Theorem 1.7. - Suppose $R$ is an admissible local ring. For every Dieudonné display $\mathscr{P}$ over $R, \mathrm{BT}(\mathscr{P})$ is a p-divisible group, i.e. $H^{i}(\mathrm{BT}(\mathscr{P}))$ vanishes for $i \neq 0$ and is a p-divisible group for $i=0$. The functor $\mathrm{BT}$ induces an equivalence of exact categories

$$
\{\text { Dieudonné displays over } R\} \cong\{\text {-divisible groups over } R\}
$$

that coincides with the equivalence in [11]. The height of $\mathrm{BT}(\mathscr{P})$ is equal to the rank of $P$, and there is a natural isomorphism $\operatorname{Lie}(\mathrm{BT}(\mathscr{P})) \cong P / Q$.

Here the additive category of Dieudonné displays is made into an exact category by declaring a short sequence to be exact if it is exact on the $P$ 's and on the $Q$ 's. Let us stress again that the only new aspect in Theorem 1.7 is the formula for the functor BT. It will be proved in the next section.

REMARK. - The functor BT is also compatible with base change, see Section 4. 


\section{Proof of the main formula}

Let $R, \mathfrak{m}, k$ be as before. We begin with recalling some definitions and results from [11] that are stated there only if $R$ is artinian, but if $\mathfrak{m}$ is nilpotent the arguments apply without change, and the general case follows by passing to the limit since Dieudonné displays over $R$ are equivalent to compatible systems of Dieudonné displays over $R / \mathfrak{m}^{n}$ for $n \geq 1$.

A Dieudonné display $\mathscr{P}$ over $R$ is called étale if $V^{\sharp}$ is an isomorphism, of multiplicative type if $F^{\sharp}$ is an isomorphism, and $V$-nilpotent or $F$-nilpotent if $V^{\sharp}$ or $F^{\sharp}$ is topologically nilpotent for the adic topology on $\mathbb{W}(R)$ defined by the ideal $\widehat{W}(\mathfrak{m})+\mathbb{I}_{R} . \mathscr{P}$ is étale if and only if $Q=P$ and of multiplicative type if and only if $Q=\mathbb{I}_{R} P$, see [11] Definitions $13 \& 14$. Etale or multiplicative or $V$-nilpotent or $F$-nilpotent Dieudonné displays over $R$ are equivalent to compatible systems of the same objects over $R / \mathfrak{m}^{n}$ for $n \geq 1$ because each of these conditions holds for $\mathscr{P}$ over $R$ if and only if it holds for the base change $\mathscr{P}_{k}$ over $k$.

By [11] Propositions 15, $16 \& 17$, there are no non-trivial homomorphisms between étale and $V$-nilpotent or between multiplicative and $F$-nilpotent Dieudonné displays in either direction, moreover for every $\mathscr{P}$ there are unique and functorial exact sequences of Dieudonné displays

$$
\begin{gathered}
0 \rightarrow \mathscr{P}^{V \text {-nil }} \rightarrow \mathscr{P} \rightarrow \mathscr{P}^{\text {et }} \rightarrow 0 \\
0 \rightarrow \mathscr{P}^{\text {mult }} \rightarrow \mathscr{P} \rightarrow \mathscr{P}^{F-\text { nil }} \rightarrow 0
\end{gathered}
$$

such that $\mathscr{P}^{\text {et }}, \mathscr{P}^{\text {mult }}, \mathscr{P}^{V \text {-nil }}, \mathscr{P}^{\text {-nil }}$ are of the designated types. The corresponding assertions for $p$-divisible groups are well-known: Let us call a $p$-divisible group $G$ over $R$ infinitesimal if $G(\bar{k})=(0)$, i.e. if $G$ is infinitesimal as a group over $\operatorname{Spf} R$. Then for every $G$ there is a unique and functorial exact sequence of $p$-divisible groups

$$
0 \rightarrow G^{\mathrm{inf}} \rightarrow G \rightarrow G^{\mathrm{et}} \rightarrow 0
$$

such that $G^{\text {et }}$ is étale and $G^{\text {inf }}$ infinitesimal, moreover by rigidity there are no non-zero homomorphisms between étale and infinitesimal $p$-divisible groups over $R$ in either direction.

The equivalence between Dieudonné displays and $p$-divisible groups in [11] is obtained by showing that $V$-nilpotent or étale Dieudonné displays are equivalent to infinitesimal or étale $p$-divisible groups, respectively, and by an explicit isomorphism $\operatorname{Ext}^{1}\left(\mathscr{P}, \mathscr{P}^{\prime}\right) \cong \operatorname{Ext}^{1}\left(G, G^{\prime}\right)$ if $\mathscr{P}$ is an étale and $\mathscr{P}^{\prime}$ a $V$-nilpotent Dieudonné display and $G, G^{\prime}$ are the associated $p$-divisible groups. In order to prove Theorem 1.7 we show that the functor BT reproduces the given equivalences in the étale and $V$-nilpotent case and that it induces the given isomorphism on $\mathrm{Ext}^{1}$.

As a preparation we define for every Dieudonné display $\mathscr{P}$ over $R$ an exact sequence of complexes in $\widetilde{\mathcal{C}}_{R}$ of the following type.

$$
0 \rightarrow Z_{\mathcal{N}}(\mathscr{P}) \rightarrow Z(\mathscr{P}) \rightarrow \bar{Z}(\mathscr{P}) \rightarrow 0 .
$$

For $S \in \mathcal{C}_{R}$ let $\bar{P}(S)=P\left(S_{\text {red }}\right)$ and $\bar{Q}(S)=Q\left(S_{\text {red }}\right)$. Then $\bar{P}$ and $\bar{Q}$ are sheaves on $\mathcal{C}_{R}^{\text {op }}$ because for every faithfully flat ring homomorphism $S \rightarrow T$ in $\mathcal{C}_{R}$ the induced homomorphism $S_{\text {red }} \rightarrow T_{\text {red }}$ is also faithfully flat (all $S_{\text {red }}$-modules are flat), and $T_{\text {red }} \otimes_{S_{\text {red }}} T_{\text {red }}$ coincides with $\left(T \otimes_{S} T\right)_{\text {red. }}$. Let

$$
\bar{Z}(\mathscr{P})=\left[\bar{Q} \stackrel{F_{1} \text {-incl }}{\longrightarrow} \bar{P}\right]
$$


and let $Z_{\mathcal{N}}(\mathscr{P})=\left[Q_{\mathcal{N}} \stackrel{F_{1} \text {-incl }}{\longrightarrow} P_{\mathcal{N}}\right]$ be the kernel of $Z(\mathscr{P}) \rightarrow \bar{Z}(\mathscr{P})$, explicitly

$$
P_{\mathcal{N}}(S)=\widehat{W}(\mathcal{N}(S)) \otimes_{\mathbb{W}(R)} P, \quad Q_{\mathcal{N}}=P_{\mathcal{N}} \cap Q .
$$

Note that (2.4) is already exact on the level of presheaves.

\subsection{The infinitesimal case}

Proposition 2.1.1. - Theorem 1.7 holds for $V$-nilpotent Dieudonné displays and infinitesimal p-divisible groups. If $\mathscr{P}$ is $V$-nilpotent and $G$ is the associated group, we have a natural quasi-isomorphism $Z(\mathscr{P}) \simeq G[-1]$.

Let us recall the proof of the infinitesimal case in [11]. To a Dieudonné display $\mathscr{P}$ over $R$ one associates a display $\mathcal{F} \mathscr{P}=\left(P^{\prime}, Q^{\prime}, F^{\prime}, F_{1}^{\prime}\right)$, where $P^{\prime}=W(R) \otimes_{\mathbb{W}(R)} P$, and $Q^{\prime}$ is the kernel of the natural map $P^{\prime} \rightarrow P / Q$. The functor $\mathcal{F}$ induces an equivalence between $V$ nilpotent Dieudonne displays and $V$-nilpotent displays. This is tautological if $R=k$; if the maximal ideal $\mathfrak{m}$ is nilpotent, the assertion follows by deformation theory; the general case by a limit argument.

On the other hand, if $\mathscr{P}=\left(P, Q, F, F_{1}\right)$ is a display over $R$ and $N$ a nilpotent (nonunitary) $R / \mathfrak{m}^{n}$-algebra for some $n$, let us write:

$$
\begin{aligned}
\widehat{P}(N) & =\widehat{W}(N) \otimes_{W(R)} P \\
\widehat{Q}(N) & =\operatorname{Ker}\left(\widehat{P}(N) \rightarrow N \otimes_{R} P / Q\right) \\
\widehat{Z}(\mathscr{P}, N) & =\left[\widehat{Q}(N) \stackrel{F_{1} \text {-incl }}{\longrightarrow} \widehat{P}(N)\right] .
\end{aligned}
$$

Then by [13] Theorem $81 \&$ Corollary 89 , the group $H^{0} \widehat{Z}(\mathscr{P}, N)$ vanishes, and the functor $N \mapsto H^{1} \widehat{Z}(\mathscr{P}, N)$ is represented by a formal group over $\operatorname{Spf} R$ that is $p$-divisible if $\mathscr{P}$ is $V$-nilpotent. By op. cit. §3.3, the functor $\mathscr{P} \mapsto H^{1} \widehat{Z}\left(\mathscr{P},{ }_{-}\right)$induces an equivalence between $V$-nilpotent displays and infinitesimal $p$-divisible groups over $R$; more precisely, Corollary 95 and a limit argument reduce this to the case $R=k$, which is covered by Proposition 102 .

If $\mathscr{P}$ is a display over $R$, let $\mathscr{Z}(\mathscr{P})$ denote the complex in $\widetilde{\mathcal{C}}_{R}$ given by $\mathscr{Z}(\mathscr{P})(S)=$ $\widehat{Z}(\mathscr{P}, \mathcal{N}(S))$ for $S \in \mathcal{C}_{R}$. For every Dieudonné display $\mathscr{P}$ over $R$ we have an obvious isomorphism

$$
Z_{\mathcal{N}}(\mathscr{P}) \cong \mathscr{Z}(\mathcal{F} \mathscr{P})
$$

It follows that $H^{0} Z_{\mathcal{N}}(\mathscr{P})$ vanishes, and the functor $H^{1} Z_{\mathcal{N}}$ defines an equivalence between $V$-nilpotent Dieudonné displays and infinitesimal $p$-divisible groups. This is the equivalence of [11]. Here $H^{1} Z_{\mathcal{N}}(\mathscr{P})$ in the sense of presheaves or sheaves is the same, i.e. the presheaf $H^{1}$ is already a sheaf.

Lemma 2.1.2. - If $\mathscr{P}$ is a V-nilpotent Dieudonné display, then $\bar{Z}(\mathscr{P})$ is acyclic.

Proof. - For $S \in \mathcal{C}_{R}$ the complex $\left[F_{1}\right.$-incl $\left.: Q_{S_{\text {red }}} \rightarrow P_{S_{\text {red }}}\right]$ is isomorphic to [id $-V:$ $\left.P_{S_{\text {red }}} \rightarrow P_{S_{\text {red }}}\right]$ where $V=F_{1}^{-1}$. Since $V$ is topologically nilpotent, id $-V$ is bijective. 
For every $K \in D\left(\widetilde{\mathcal{C}}_{R}\right)$ the obvious homomorphisms of complexes

$$
\mathbb{Q}_{p} / \mathbb{Z}_{p} \stackrel{\simeq}{\longleftarrow}\left[\mathbb{Z} \rightarrow \mathbb{Z}\left[\frac{1}{p}\right]\right] \longrightarrow \mathbb{Z}[1]
$$

(where $\simeq$ means quasi-isomorphism) induce a homomorphism $\pi_{K}: K \otimes^{L} \mathbb{Q}_{p} / \mathbb{Z}_{p} \rightarrow K[1]$. It is an isomorphism if all local sections of $H^{*} K$ are annihilated by powers of $p$.

Proof of Proposition 2.1.1. - By the above discussion and Lemma 2.1.2 we have an equivalence $\mathscr{P} \mapsto G$ between $V$-nilpotent Dieudonné displays and infinitesimal $p$-divisible groups such that $Z(\mathscr{P}) \simeq Z_{\mathcal{N}}(\mathscr{P}) \simeq G[-1]$. The isomorphism $\pi_{G[-1]}$ then gives $\mathrm{BT}(\mathscr{P}) \cong G$. By [13] (158) there is a natural isomorphism $\operatorname{Lie}(G) \cong P / Q$.

ExAmple. - The Dieudonné display associated to $\widehat{\mathbb{G}}_{m}$ is

$$
\mathscr{G}_{m}=\left(\mathbb{W}(R), \mathbb{I}_{R}, f, v^{-1}\right)
$$

where $v^{-1}$ is the inverse of the bijective homomorphism $v: \mathbb{W}(R) \rightarrow \mathbb{I}_{R}$. In fact, for every nilpotent $R / \mathfrak{m}^{n}$-algebra $N$ there is an exact sequence

$$
0 \rightarrow \widehat{W}(N) \stackrel{1-v}{\longrightarrow} \widehat{W}(N) \stackrel{\text { hex }}{\longrightarrow} \widehat{\mathbb{G}}_{m}(N) \rightarrow 0
$$

where hex is given by the Artin-Hasse exponential evaluated at $t=1$, see [13] p. 108, hence $\operatorname{BT}\left(\mathscr{G}_{m}\right) \cong H^{1} Z_{\mathcal{N}}\left(\mathscr{G}_{m}\right) \cong \widehat{\mathbb{G}}_{m}$.

\subsection{The étale case}

If $G$ is an étale $p$-divisible group over $R$, let $T_{p} G=\lim G\left[p^{n}\right]$ in $\widetilde{\mathcal{C}}_{R}$. The obvious sequences $0 \rightarrow T_{p} G \rightarrow T_{p} G \rightarrow G\left[p^{n}\right] \rightarrow 0$ are exact because arbitrary ind-étale coverings exist in $\mathcal{C}_{R}$, and they give an isomorphism $T_{p} G \otimes^{L} \mathbb{Q}_{p} / \mathbb{Z}_{p} \cong G$.

Proposition 2.2.1. - Theorem 1.7 holds in the étale case. If $G$ is the étale p-divisible group associated to an étale Dieudonné display $\mathscr{P}$, we have a natural quasi-isomorphism $T_{p} G \simeq Z(\mathscr{P})$.

Note that an étale Dieudonné display is the same as a pair $\left(P, F_{1}\right)$, where $P$ is a finitely generated free $\mathbb{W}(R)$-module and $F_{1}^{\sharp}: P^{(1)} \rightarrow P$ an isomorphism; we have $Q=P$ and $F=p F_{1}$. The complex $Z(\mathscr{P})$ takes the form $\left[F_{1}-\right.$ id $\left.: P \rightarrow P\right]$.

Again we have to recall the equivalence $\mathscr{P} \mapsto G$ between étale Dieudonné displays and étale $p$-divisible groups from [11]. By op. cit. Theorem 5, étale Dieudonné displays over $R$ and over $k$ are equivalent. The analogous assertion for étale $p$-divisible groups and for truncated étale $p$-divisible groups is well-known. Hence it suffices to define the equivalence $\mathscr{P} \mapsto G$ over $k$; there it is given by the isomorphism of $\operatorname{Gal}(\bar{k} / k)$-modules

$$
T_{p} G(\bar{k})=\left(W(\bar{k}) \otimes_{W(k)} P\right)^{F_{1}=\mathrm{id}} .
$$

Let us reformulate this a little. Over every ring $A$ of characteristic $p$, truncated étale $p$-divisible groups of level $n$ are equivalent to pairs $(M, \Phi)$, where $M$ is a finitely generated projective $W_{n}(A)$-module and $\Phi: M^{(1)} \rightarrow M$ is an isomorphism. The group associated to $(M, \Phi)$ is the sheaf that maps an $A$-algebra $B$ to $\left(M \otimes_{A} B\right)^{\Phi=\text { id }}$. 
Assume now that $\mathscr{P} \mapsto G$ as above and that $p R=0$. Then the pair $(M, \Phi)$ associated to the truncated étale group $G\left[p^{n}\right]$ is naturally isomorphic to $\left(W_{n}(R) \otimes_{\mathbb{W}(R)} P, F_{1}\right)$. In fact, to prove this one may pass to $k$, where the assertion follows from (2.8). For $S \in \mathcal{C}_{R}$ we get

$$
G\left[p^{n}\right](S)=\left(W_{n}(S) \otimes_{\mathbb{W}(R)} P\right)^{F_{1}=\mathrm{id}} .
$$

Lemma 2.2.2. - If $\mathscr{P}$ is an étale Dieudonné display, then $Z_{\mathcal{N}}(\mathscr{P})$ is acyclic.

Proof. - Since $F_{1}: P \rightarrow P$ is $f$-linear, the induced map $F_{1}: P_{\mathcal{N}} \rightarrow P_{\mathcal{N}}$ is elementwise nilpotent, so $F_{1}-$ id $: P_{\mathcal{N}} \rightarrow P_{\mathcal{N}}$ is bijective.

Proof of Proposition 2.2.1. - If $\mathscr{P}$ is an étale Dieudonné display over $R$ and $G$ is the associated étale $p$-divisible group, the sheaves $H^{i} \bar{Z}(\mathscr{P})$ are computed as follows. For $S \in \mathcal{C}_{R}$ we have, using (2.9),

$$
\begin{aligned}
H^{0} \bar{Z}(\mathscr{P})(S) & =\lim ^{\lim }\left(W_{n}\left(S_{\text {red }}\right) \otimes_{\mathbb{W}(R)} P\right)^{F_{1}=\mathrm{id}} \\
& =\lim _{\longleftarrow} G\left[p^{n}\right]\left(S_{\text {red }}\right)=\varliminf_{\longleftarrow}^{\lim } G\left[p^{n}\right](S)=T_{p} G(S) .
\end{aligned}
$$

The sheaf $H^{1} \bar{Z}(\mathscr{P})$ vanishes because every element of $P\left(S_{\text {red }}\right)$ has an inverse image under $F_{1}-$ id after passing to an ind-étale covering of $S_{\text {red }}$, which lifts to an ind-étale covering of $S$. By Lemma 2.2.2 we obtain $Z(\mathscr{P}) \simeq \bar{Z}(\mathscr{P}) \simeq T_{p} G$, hence $\mathrm{BT}(\mathscr{P}) \cong G$.

\subsection{Calculation of extensions}

For an étale $p$-divisible group $H$ over $R$ there is a natural exact sequence in $\widetilde{\mathcal{C}}_{R}$

$$
0 \rightarrow T_{p} H \rightarrow T_{p} H \otimes \mathbb{Z}\left[\frac{1}{p}\right] \rightarrow H \rightarrow 0 .
$$

If $G$ is another $p$-divisible group over $R$, we obtain a connecting homomorphism:

$$
\delta: \operatorname{Hom}\left(T_{p} H, G\right) \rightarrow \operatorname{Ext}^{1}(H, G) .
$$

REMARK. - We have an isomorphism $\operatorname{Hom}\left(T_{p} H, G\right) \cong \underset{\lim }{\longrightarrow} \operatorname{Hom}\left(H\left[p^{n}\right], G\right)$ because $T_{p} H$

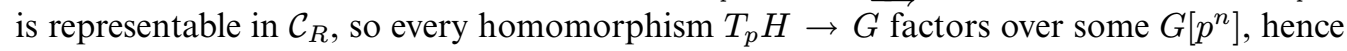
also over $H\left[p^{n}\right]$. Using that isomorphism, $\delta$ can be defined by the obvious exact sequences $0 \rightarrow H\left[p^{n}\right] \rightarrow H \rightarrow H \rightarrow 0$ instead of (2.10).

Proposition 2.3.1. - If $H$ is étale and $G$ is infinitesimal, then $\delta$ is bijective.

This is [11] Proposition 19. See Proposition A.1 for a more general statement.

We turn to extensions of Dieudonné displays. Let $\mathscr{P}^{\text {et }}$ be an étale and $\mathscr{P}^{\text {nil }}$ a $V$-nilpotent Dieudonné display over $R$ and let $H=\mathrm{BT}\left(\mathscr{P}^{\mathrm{et}}\right)$ and $G=\mathrm{BT}\left(\mathscr{P}^{\text {nil }}\right)$ be the associated $p$-divisible groups. For every extension of Dieudonné displays

$$
0 \rightarrow \mathscr{P}^{\text {nil }} \rightarrow \mathscr{P} \rightarrow \mathscr{P}^{\text {et }} \rightarrow 0
$$

the resulting exact sequence of complexes

$$
0 \rightarrow Z\left(\mathscr{P}^{\text {nil }}\right) \rightarrow Z(\mathscr{P}) \rightarrow Z\left(\mathscr{P}^{\text {et }}\right) \rightarrow 0
$$

gives rise to a connecting homomorphism

$$
T_{p} H \cong H^{0} Z\left(\mathscr{P}^{\mathrm{et}}\right) \rightarrow H^{1} Z\left(\mathscr{P}^{\mathrm{nil}}\right) \cong G
$$


where the outer isomorphisms are provided by Propositions 2.1.1 \& 2.2.1. This construction defines a homomorphism

$$
\gamma: \operatorname{Ext}^{1}\left(\mathscr{P}^{\mathrm{et}}, \mathscr{P}^{\mathrm{nil}}\right) \rightarrow \operatorname{Hom}\left(T_{p} H, G\right) .
$$

Proposition 2.3.2. - The homomorphism $\gamma$ is bijective.

This is a reformulation of [11] Proposition 18 and its proof. The equivalence between Dieudonné displays and $p$-divisible groups in op. cit. is defined by the composite isomorphism $\delta \gamma: \operatorname{Ext}^{1}\left(\mathscr{P}^{\text {et }}, \mathscr{P}^{\text {nil }}\right) \rightarrow \operatorname{Ext}^{1}(H, G)$.

Proof of Theorem 1.7. - By Propositions 2.1.1 \& 2.2.1 we know that $\mathrm{BT}(\mathscr{P})$ is a $p$-divisible group of the correct height if $\mathscr{P}$ is étale or $V$-nilpotent. The same is true for general $\mathscr{P}$ because the exact sequence (2.1) gives rise to a distinguished triangle $\mathrm{BT}\left(\mathscr{P}^{V \text {-nil }}\right) \rightarrow \mathrm{BT}(\mathscr{P}) \rightarrow \mathrm{BT}\left(\mathscr{P}^{\mathrm{et}}\right) \rightarrow^{+}$. Similarly we see that $\mathrm{BT}$ preserves arbitrary exact sequences.

The main point to be shown is that for $H=\mathrm{BT}\left(\mathscr{P}^{\mathrm{et}}\right)$ and $G=\mathrm{BT}\left(\mathscr{P}^{\text {nil }}\right)$ as above, the homomorphism $\operatorname{Ext}^{1}\left(\mathscr{P}^{\text {et }}, \mathscr{P}^{\text {nil }}\right) \rightarrow \operatorname{Ext}^{1}(H, G)$ induced by BT coincides with the isomorphism $\delta \gamma$.

Let us first describe the action of BT on $\operatorname{Ext}^{1}$. Let $T=T_{p} H$. If an extension (2.11) is given, then using the quasi-isomorphisms $Z\left(\mathscr{P}^{\text {nil }}\right) \simeq G[-1]$ and $Z\left(\mathscr{P}^{\text {et }}\right) \simeq T$, the corresponding extension (2.12) determines the following distinguished triangle in $D\left(\widetilde{\mathcal{C}}_{R}\right)$, where $g$ is the negative of the connecting homomorphism (2.13).

$$
G[-1] \rightarrow Z(\mathscr{P}) \rightarrow T \stackrel{g}{\rightarrow} G .
$$

The functor BT applied to (2.11) results in (2.14) $\otimes^{L} \mathbb{Q}_{p} / \mathbb{Z}_{p}$, which under the identification $\pi_{G[-1]}: G[-1] \otimes^{L} \mathbb{Q}_{p} / \mathbb{Z}_{p} \cong G$ induced by (2.5) takes the following form.

$$
G \rightarrow \mathrm{BT}(\mathscr{P}) \rightarrow H \stackrel{g^{\prime}}{\rightarrow} G[1] .
$$

Here $g^{\prime}$ is the composition

$$
H \cong T \otimes^{L} \mathbb{Q}_{p} / \mathbb{Z}_{p} \stackrel{g \otimes \mathrm{id}}{\longrightarrow} G \otimes^{L} \mathbb{Q}_{p} / \mathbb{Z}_{p} \stackrel{-\pi_{G}}{\longrightarrow} G[1]
$$

because $-\pi_{G}$ gets identified with $\pi_{G[-1]}[1]$ under the natural isomorphism of $G \otimes \otimes^{L} \mathbb{Q}_{p} / \mathbb{Z}_{p}$ with $G[-1] \otimes{ }^{L} \mathbb{Q}_{p} / \mathbb{Z}_{p}[1]$, the sign arising from the transposition automorphism of $\mathbb{Z}[1] \otimes \mathbb{Z}[1]$, which is - id.

Consider now $\delta \gamma$. Since the extension (2.10) corresponds to the distinguished triangle

$$
T \rightarrow T \otimes \mathbb{Z}\left[\frac{1}{p}\right] \rightarrow T \otimes \mathbb{Q}_{p} / \mathbb{Z}_{p} \stackrel{\pi_{T}}{\longrightarrow} T[1]
$$

and since $g$ is the negative of (2.13), the image of (2.11) under $\delta \gamma$ corresponds to a triangle of the form (2.15) with $g^{\prime}=-g[1] \pi_{T}$. It remains to show that $g[1] \pi_{T}=\pi_{G}(g \otimes \mathrm{id})$, which is immediate from the definitions.

Finally, the inverse functor of BT preserves exact sequences because this is true over $k$, and a short sequence of Dieudonné displays over $R$ is exact if and only if it is exact over $k$ by Nakayama's lemma. The existence of a natural isomorphism $\operatorname{Lie}(\operatorname{BT}(\mathscr{P})) \cong P / Q$ follows from the $V$-nilpotent case since $\operatorname{Lie}(\mathrm{BT}(\mathscr{P}))=\operatorname{Lie}\left(\mathrm{BT}\left(\mathscr{P}{ }^{\text {nil }}\right)\right)$ and $P / Q=P^{\text {nil }} / Q^{\text {nil }}$.

A $V$-nilpotent and $F$-nilpotent Dieudonné display is called bi-nilpotent. 
COROLlaRY 2.4. - The group $\mathrm{BT}(\mathscr{P})$ is étale or of multiplicative type or bi-infinitesimal if and only if $\mathscr{P}$ is étale or of multiplicative type or bi-nilpotent, respectively.

Proof. - Since a $p$-divisible group over $R$ is étale or of multiplicative type if and only if its dimension is equal to zero or its height, the étale and multiplicative cases of the corollary are a direct consequence of the last assertion of Theorem 1.7. The third case follows because biinfinitesimal groups and bi-nilpotent Dieudonné displays are both characterised by having neither non-trivial sub-objects of multiplicative type nor étale quotients.

REMARK. - The proof of Corollary 2.4 requires only that the functor BT is an equivalence over $k$, which is classical, because the relevant properties of Dieudonné displays and $p$-divisible groups over $R$ depend only on their fibres over $k$.

We conclude this section with a remark on the topology chosen on $\mathcal{C}_{R}^{\text {op }}$.

First let us note that $p$-divisible groups over $R$ form a full exact subcategory of the category of sheaves on $\mathcal{C}_{R}^{\text {op }}$ for the étale or (ind-étale) topology, i.e. every short exact sequence $0 \rightarrow H \rightarrow E \rightarrow G \rightarrow 0$ of $p$-divisible groups gives rise to an exact sequence of étale sheaves. Indeed, recall that according to [7] Lemma 10.12, every $H$-torsor is formally smooth. Thus the sequence admits a set-theoretical section if $G$ is infinitesimal, or if $G$ is étale and $H$ is infinitesimal since then it splits over $k$. By using the functorial decomposition (2.3) if follows that the sequence of étale sheaves is exact. However, multiplication by $p$ is a surjective endomorphism of the étale sheaf given by a $p$-divisible group only if the group is étale.

For a Dieudonné display $\mathscr{P}$ over $R$ let

$$
Y(\mathscr{P})=\left[Q \stackrel{F_{1}-1}{\longrightarrow} P\right] \otimes\left[\mathbb{Z} \rightarrow \mathbb{Z}\left[\frac{1}{p}\right]\right]
$$

as a complex of presheaves on $\mathcal{C}_{R}^{\text {op }}$ concentrated in degrees $-1,0,1$. This is in fact a complex of flat sheaves as $P(S) \otimes \mathbb{Z}\left[\frac{1}{p}\right]=Q(S) \otimes \mathbb{Z}\left[\frac{1}{p}\right]=W\left(S_{\text {red }}\right)\left[\frac{1}{p}\right] \otimes_{\mathbb{W}(R)} P$ for $S \in \mathcal{C}_{R}$, which clearly is a sheaf. The following refinement of Theorem 1.7 is an immediate consequence of its proof.

COROLlary 2.5. - The ind-étale cohomology sheaf $H^{i}(Y(\mathscr{P}))$ is naturally isomorphic to $\mathrm{BT}(\mathscr{P})$ for $i=0$ and vanishes for $i \neq 0$.

\section{Duality}

Let $R, \mathfrak{m}, k$ be as before. In order to define the dual of a Dieudonné display we need the following notion of bilinear forms. Recall the definition of $\mathscr{G}_{m}$ in (2.6). If $\mathscr{P}$ and $\mathscr{P}^{\prime}$ are Dieudonné displays over $R$, a bilinear form $\alpha: \mathscr{P} \times \mathscr{P}^{\prime} \rightarrow \mathscr{G}_{m}$ is by definition a $\mathbb{W}(R)$ bilinear map $\alpha: P \times P^{\prime} \rightarrow \mathbb{W}(R)$ such that

$$
v\left(\alpha\left(F_{1} x, F_{1}^{\prime} x^{\prime}\right)\right)=\alpha\left(x, x^{\prime}\right)
$$

for $x \in Q$ and $x^{\prime} \in Q^{\prime}$. This also implies for $y \in P, y^{\prime} \in P^{\prime}$, and $x, x^{\prime}$ as before:

$$
\begin{aligned}
\alpha\left(F_{1} x, F^{\prime} y^{\prime}\right) & =f\left(\alpha\left(x, y^{\prime}\right)\right) \\
\alpha\left(F y, F_{1}^{\prime} x^{\prime}\right) & =f\left(\alpha\left(y, x^{\prime}\right)\right) \\
\alpha\left(F y, F^{\prime} y^{\prime}\right) & =p f\left(\alpha\left(y, y^{\prime}\right)\right) .
\end{aligned}
$$


Let $\operatorname{Bil}\left(\mathscr{P} \times \mathscr{P}^{\prime}, \mathscr{G}_{m}\right)$ denote the abelian group of bilinear forms.

Definition 3.1. - For every Dieudonné display $\mathscr{P}$ over $R$ the contravariant functor $\mathscr{P}^{\prime} \mapsto \operatorname{Bil}\left(\mathscr{P}^{\prime} \times \mathscr{P}, \mathscr{G}_{m}\right)$ is represented by a Dieudonné display $\mathscr{P}^{t}$, called the dual of $\mathscr{P}$.

This is analogous to the case of displays, [13] Definition 19. Let us make the definition of $\mathscr{P}^{t}$ more explicit, which can also be used to show that $\mathscr{P}^{t}$ exists. For a $\mathbb{W}(R)$-module $M$ let $M^{\vee}$ be the module of linear maps $M \rightarrow \mathbb{W}(R)$. Then

$$
\mathscr{P}^{t}=\left(P^{\vee}, \widetilde{Q}, F^{\prime}, F_{1}^{\prime}\right)
$$

where $\widetilde{Q}=\left\{x \in P^{\vee} \mid x(Q) \subseteq \mathbb{I}_{R}\right\}$. If $P=L \oplus T$ is a normal decomposition, i.e. $Q=$ $L \oplus \mathbb{I}_{R} T$, accordingly $P^{\vee}=L^{\vee} \oplus T^{\vee}$ and $\widetilde{Q}=\mathbb{I}_{R} L^{\vee} \oplus T^{\vee}$, the formulas (3.1) to (3.4) determine that the composition

$$
\left(L^{\vee}\right)^{(1)} \oplus\left(T^{\vee}\right)^{(1)} \stackrel{\left(F^{\prime}, F_{1}^{\prime}\right)^{\sharp}}{\longrightarrow} P^{\vee} \stackrel{\left(F_{1}^{\sharp}, F^{\sharp}\right)^{\vee}}{\longrightarrow}\left(L^{(1)}\right)^{\vee} \oplus\left(T^{(1)}\right)^{\vee}
$$

is the tautological isomorphism, i.e. when passing from $\mathscr{P}$ to $\mathscr{P} t$, the matrix of $\left(F_{1}^{\sharp}, F^{\sharp}\right)$ gets transposed, inverted, and the roles of $L, T$ exchanged.

Since $V^{\sharp}\left(F_{1} x\right)=1 \otimes x$ by definition, (3.2) and (3.3) imply that $F^{\prime \sharp}=\left(V^{\sharp}\right)^{\vee}$ and $V^{\sharp \sharp}=\left(F^{\sharp}\right)^{\vee}$, thus dualising interchanges étale and multiplicative as well as $V$-nilpotent and $F$-nilpotent Dieudonné displays.

\section{Definition of the duality homomorphism}

In the remainder of this article, let always $G=\mathrm{BT}(\mathscr{P})$ and $G^{\prime}=\mathrm{BT}\left(\mathscr{P}^{\prime}\right)$ and, by a slight abuse of notation, $G^{t}=\mathrm{BT}\left(\mathscr{P}^{t}\right)$. For arbitrary $\mathscr{P}$ and $\mathscr{P}^{\prime}$ we want to construct a functorial homomorphism

$$
\psi: \operatorname{Bil}\left(\mathscr{P}^{\prime} \times \mathscr{P}, \mathscr{G}_{m}\right) \rightarrow \operatorname{Ext}^{1}\left(G^{\prime} \otimes^{L} G, \widehat{\mathbb{G}}_{m}\right) .
$$

Given a bilinear form $\alpha: \mathscr{P}^{\prime} \times \mathscr{P} \rightarrow \mathscr{G}_{m}$ let us first define a homomorphism of complexes $\gamma: Z\left(\mathscr{P}^{\prime}\right) \otimes Z(\mathscr{P}) \rightarrow Z\left(\mathscr{G}_{m}\right)$, which is equivalent to homomorphisms $\gamma_{0}$ and $\gamma_{1}$ forming the following commutative diagram, where $\varphi=F_{1}-$ id and $\varphi^{\prime}=F_{1}^{\prime}-$ id.

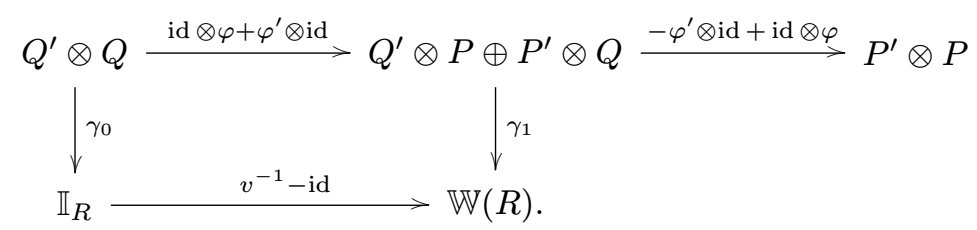

We let $\gamma_{0}=\alpha$, and there are two choices for $\gamma_{1}$ : either $\gamma_{1}\left(q^{\prime} \otimes p+p^{\prime} \otimes q\right)=\alpha\left(q^{\prime}, p\right)+\alpha\left(p^{\prime}, F_{1} q\right)$ or $\gamma_{1}\left(q^{\prime} \otimes p+p^{\prime} \otimes q\right)=\alpha\left(F_{1}^{\prime} q^{\prime}, p\right)+\alpha\left(p^{\prime}, q\right)$. The two resulting maps $\gamma$ are homotopic via the homotopy consisting of $\alpha: P^{\prime} \otimes P \rightarrow \mathbb{W}(R)$ in top degree and zero in lower degrees, in particular the homomorphism in $D\left(\widetilde{\mathcal{C}}_{R}\right)$

$$
\gamma^{L}: Z\left(\mathscr{P}^{\prime}\right) \otimes^{L} Z(\mathscr{P}) \stackrel{\text { can }}{\longrightarrow} Z\left(\mathscr{P}^{\prime}\right) \otimes Z(\mathscr{P}) \stackrel{\gamma}{\rightarrow} Z\left(\mathscr{G}_{m}\right)
$$


is independent of the choice. From $\gamma^{L}$ we obtain the following morphism in $D\left(\widetilde{\mathcal{C}}_{R}\right)$ that can be viewed as an element of $\operatorname{Ext}^{1}\left(G^{\prime} \otimes^{L} G, \widehat{\mathbb{G}}_{m}\right)$; by definition this is $\psi(\alpha)$.

$$
\begin{aligned}
G^{\prime} \otimes^{L} G \cong Z\left(\mathscr{P}^{\prime}\right) \otimes^{L} Z(\mathscr{P}) \otimes^{L} \mathbb{Q}_{p} / \mathbb{Z}_{p} \otimes^{L} \mathbb{Q}_{p} / \mathbb{Z}_{p} \\
\quad \stackrel{\gamma^{L} \otimes \mathrm{id} \otimes \mathrm{id}}{\longrightarrow} Z\left(\mathscr{G}_{m}\right) \otimes^{L} \mathbb{Q}_{p} / \mathbb{Z}_{p} \otimes^{L} \mathbb{Q}_{p} / \mathbb{Z}_{p} \cong \widehat{\mathbb{G}}_{m} \otimes^{L} \mathbb{Q}_{p} / \mathbb{Z}_{p} \stackrel{(2.5)}{\cong} \widehat{\mathbb{G}}_{m}[1] .
\end{aligned}
$$

A direct computation of $G^{\prime} \otimes^{L} G$ yields the following isomorphism, explained in detail in SGA 7, VIII, 1.3

$$
\operatorname{Ext}^{1}\left(G^{\prime} \otimes L, \widehat{\mathbb{G}}_{m}\right) \cong \operatorname{Hom}\left(G^{\prime}, G^{\vee}\right)
$$

Definition 3.2. - The duality homomorphism $\Psi: G^{t} \rightarrow G^{\vee}$ is the image of the canonical bilinear form $\mathscr{P}^{t} \times \mathscr{P} \rightarrow \mathscr{G}_{m}$ under $\psi$ composed with (3.5).

REMARK. - The homomorphism $\Psi$ is compatible with base change, see section 4 .

Naturality of $\psi$ gives the next commutative diagram, which is just an explication of the fact that $\psi$ and $\Psi$ are equivalent by the Yoneda lemma.

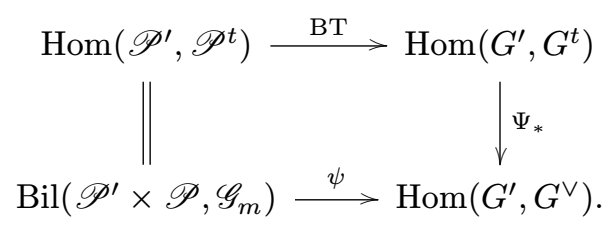

Here by Theorem 1.7 we know (but shall not use) that BT is bijective.

Lemma 3.3. - There is the following anti-commutative diagram:

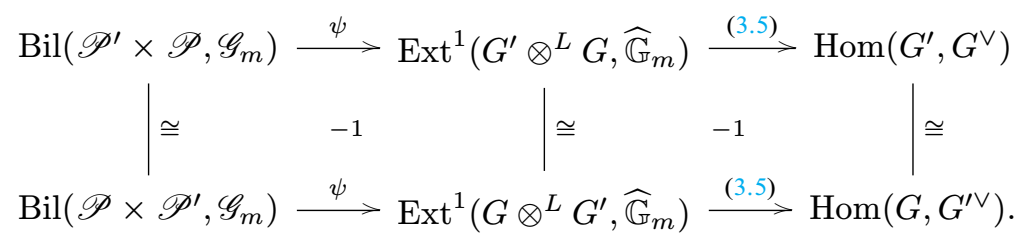

Proof. - The right hand square is SGA 7, VIII, Proposition 2.2.11. The left hand square follows from the definitions, the sign resulting from the transposition automorphism of $\mathbb{Q}_{p} / \mathbb{Z}_{p} \otimes^{L} \mathbb{Q}_{p} / \mathbb{Z}_{p}$, which is - id.

In particular, a skew symmetric bilinear form $\mathscr{P} \times \mathscr{P} \rightarrow \mathscr{G}_{m}$ gives a symmetric biextension and an anti-symmetric homomorphism $G \rightarrow G^{\vee}$.

Theorem 3.4. - For every Dieudonné display $\mathscr{P}$ over an admissible local ring $R$ the duality homomorphism $\Psi: \mathrm{BT}\left(\mathscr{P}^{t}\right) \rightarrow \mathrm{BT}(\mathscr{P})^{\vee}$ is an isomorphism. 
By Theorem 1.7 this implies that $\psi$ is an isomorphism as well.

For the proof of Theorem 3.4 we begin with a number of reductions. Using the decompositions (2.1) and (2.2) we may assume that $\mathscr{P}$ is étale or of multiplicative type or bi-nilpotent. By Lemma 3.3 the assertion for $\mathscr{P}$ is equivalent to the assertion for $\mathscr{P}^{t}$, so the multiplicative case can be omitted. Since a homomorphism of $p$-divisible groups over $R$ is an isomorphism if and only if it is an isomorphism over $\bar{k}$, we may assume that $R$ is an algebraically closed field.

Proof of Theorem 3.4 in the étale case. - We may assume that $G=\mathbb{Q}_{p} / \mathbb{Z}_{p}$, accordingly $\mathscr{P}=(\mathbb{W}(R), \mathbb{W}(R), p f, f)$ and $\mathscr{P}^{t}=\mathscr{G}_{m}$. In order that $\Psi: \widehat{\mathbb{G}}_{m} \rightarrow\left(\mathbb{Q}_{p} / \mathbb{Z}_{p}\right)^{\vee}$ is an isomorphism it suffices that it is not divisible by $p$. To get $\Psi$ we trace the definition of $\psi$ applied to the natural bilinear form $\alpha: \mathscr{G}_{m} \times \mathscr{P} \rightarrow \mathscr{G}_{m}$. Under the quasi-isomorphisms $Z\left(\mathscr{G}_{m}\right) \simeq \widehat{\mathbb{G}}_{m}[-1]$ and $T_{p}\left(\mathbb{Q}_{p} / \mathbb{Z}_{p}\right) \simeq Z(\mathscr{P})$ the homomorphism $\gamma^{L}$ (defined by the first choice for $\gamma_{1}$ ) gets identified with the tautological isomorphism $\widehat{\mathbb{G}}_{m}[-1] \otimes^{L} T_{p}\left(\mathbb{Q}_{p} / \mathbb{Z}_{p}\right) \cong \widehat{\mathbb{G}}_{m}[-1]$. Hence $\psi(\alpha)$ is the isomorphism $\widehat{\mathbb{G}}_{m} \otimes^{L} \mathbb{Q}_{p} / \mathbb{Z}_{p} \cong \widehat{\mathbb{G}}_{m}[1]$ induced by $(2.5)$, in particular $\psi(\alpha)$ is not divisible by $p$, so the same is true for $\Psi$, its image under (3.5). Note that we did not use the definition of (3.5). We leave it to the reader to determine whether $\Psi$ is the identity or its negative.

The bi-nilpotent case relies on the following theorem of Cartier. For any ring $A$ let $\widehat{W}$ be the functor on $A$-algebras $\widehat{W}(B)=\widehat{W}(\mathcal{N}(B))$. Then by [3] the bilinear maps

$$
W(A) \times \widehat{W}(B) \stackrel{\text { mult }}{\longrightarrow} \widehat{W}(B) \stackrel{\text { hex }}{\longrightarrow} \widehat{\mathbb{G}}_{m}(B)
$$

induce an isomorphism $W(A) \cong \operatorname{Hom}\left(\widehat{W}, \widehat{\mathbb{G}}_{m}\right)$, thus an isomorphism of sheaves on $\mathcal{C}_{R}^{\text {op }}$

$$
W \cong \underline{\operatorname{Hom}}\left(\widehat{W}, \widehat{\mathbb{G}}_{m}\right)
$$

because passing from functors on all $R$-algebras to functors on $\mathcal{C}_{R}$ makes no difference as $\widehat{W}$ is the direct limit of functors that are represented by rings in $\mathcal{C}_{R}$. Let $W[f]$ be the kernel of $f: W \rightarrow W$. Since $f$ corresponds to the dual of $v$ under (3.6) and the cokernel of $v: \widehat{W} \rightarrow \widehat{W}$ is $\widehat{\mathbb{G}}_{a}$, we deduce an isomorphism

$$
W[f] \cong \operatorname{Hom}\left(\widehat{\mathbb{G}}_{a}, \widehat{\mathbb{G}}_{m}\right) .
$$

Lemma 3.5. - The Frobenius homomorphism $f: W \rightarrow W$ defines a surjective homomorphism of flat sheaves on $\mathcal{C}_{R}^{\text {op }}$.

Proof. - As a functor on rings, $W$ is represented by the $\operatorname{ring} B=\mathbb{Z}\left[X_{0}, X_{1}, \ldots\right]$ and $f$ by a faithfully flat ring homomorphism $f^{\sharp}: B \rightarrow B$. In fact, since the truncated Frobenius $f_{n}: W_{n+1} \rightarrow W_{n}$ is a group homomorphism which is surjective on geometric points, its fibres are one-dimensional. Hence $f_{n}^{\sharp}: \mathbb{Z}\left[X_{0}, \ldots, X_{n}\right] \rightarrow \mathbb{Z}\left[X_{0}, \ldots, X_{n+1}\right]$ is faithfully flat by [6] Theorem 23.1, so $f^{\sharp}=\lim _{\longrightarrow} f_{n}^{\sharp}$ is faithfully flat. It remains to show that if $B \rightarrow S$ is a ring homomorphism with $S \in \overrightarrow{\mathcal{C}_{R}}$, then $B \otimes_{f^{\sharp}, B} S$ lies in $\mathcal{C}_{R}$. This can be deduced from the relation $f^{\sharp}\left(X_{i}\right) \equiv X_{i}^{p}$ modulo $p$. 
Proof of Theorem 3.4 in the bi-nilpotent case. - Assume that $\mathscr{P}$ is bi-nilpotent. Since $\Psi$ is a homomorphism of $p$-divisible groups of the same height, it suffices that $\Psi$ is injective, or even that $\Delta \circ \Psi$ is injective for some homomorphism

$$
\Delta: G^{\vee} \rightarrow \underline{\operatorname{Ext}}^{1}\left(G, \widehat{\mathbb{G}}_{m}\right) .
$$

Let $\mathcal{F} \mathscr{P}^{t}=\left(\widetilde{P^{t}}, \widetilde{Q}^{t}, F^{t}, F_{1}^{t}\right)$ be the display associated to $\mathscr{P}^{t}$ and view $\widetilde{P}^{t}, \widetilde{Q}^{t}$ as sheaves on $\mathcal{C}_{R}^{\text {op }}$. From (3.6) we get an isomorphism $u: \widetilde{P}^{t} \cong \underline{\operatorname{Hom}}\left(P_{\mathcal{N}}, \widehat{\mathbb{G}}_{m}\right)$. We claim that $\Delta$ can be chosen such that we have the following commutative diagram in $\widetilde{\mathcal{C}}_{R}$ with exact rows, where $i: Q_{\mathcal{N}} \rightarrow P_{\mathcal{N}}$ denotes the inclusion. The diagram is similar to [13] (223) in a different technical context.

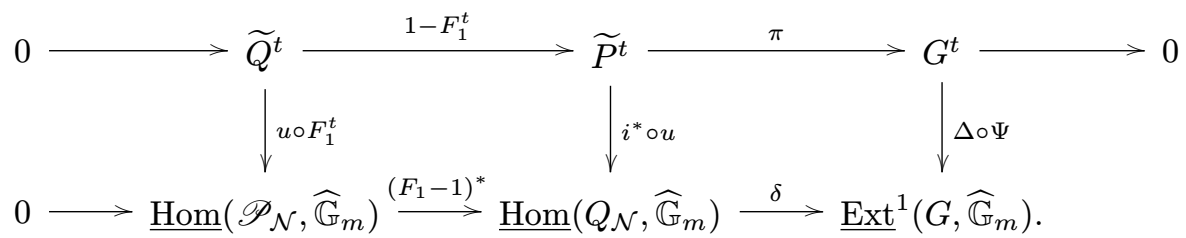

Before proving the claim, let us apply the snake lemma and deduce that $\Delta \circ \Psi$ is injective as required. Since $u$ is an isomorphism it suffices that $F_{1}^{t}$ is surjective and $u \circ\left(1-F_{1}^{t}\right)$, or equivalently $u$, induces an isomorphism $\operatorname{Ker}\left(F_{1}^{t}\right) \cong \operatorname{Ker}\left(i^{*}\right)$. In terms of a normal decomposition $P=L \oplus T$ and the induced normal decomposition $\widetilde{P}^{t}=\widetilde{T}^{t} \oplus \widetilde{L}^{t}$, the homomorphism $F_{1}^{t}$ is equal to

$$
\begin{aligned}
I_{R} \widetilde{L}^{t} \oplus \widetilde{T}^{t} & \longrightarrow\left(\widetilde{L}^{t}\right)^{(1)} \oplus\left(\widetilde{T}^{t}\right)^{(1)} \stackrel{\left(F^{t}, F_{1}^{t}\right)^{\sharp}}{\longrightarrow} \widetilde{P}^{t} \\
\left(v\left(w_{1}\right) l, w_{2} t\right) & \longmapsto\left(w_{1} \otimes l, f\left(w_{2}\right) \otimes t\right)
\end{aligned}
$$

where $\left(F^{t}, F_{1}^{t}\right)^{\sharp}$ is an isomorphism. By Lemma 3.5 it follows that $F_{1}^{t}$ is surjective, moreover $\operatorname{Ker}\left(F_{1}^{t}\right)=W[f] \otimes_{W} \widetilde{T}^{t}$. Let $\widehat{V}$ be the formal completion of the vector group $P / Q \cong T / \mathbb{I}_{R} T$. Then $\operatorname{Ker}\left(i^{*}\right) \cong \underline{\operatorname{Hom}}\left(\widehat{V}, \widehat{\mathbb{G}}_{m}\right)$, and (3.7) finishes the proof.

Let us now look at those parts of (3.8) that do not involve $\Delta$. It is straightforward that the left hand square commutes, using that $\operatorname{hex}(v(w))=\operatorname{hex}(w)$ for $w \in \widehat{W}$ according to (2.7). Consider the following complexes of sheaves in $\widetilde{\mathcal{C}}_{R}$ concentrated in degrees 0,1 .

$$
Z=\left[Q_{\mathcal{N}} \stackrel{F_{1}-1}{\longrightarrow} P_{\mathcal{N}}\right], \quad Z^{t}=\left[\widetilde{Q}^{t} \stackrel{F_{1}^{t}-1}{\longrightarrow} \widetilde{P}^{t}\right]
$$

Since $\mathscr{P}^{t}$ is $V$-nilpotent, $Z\left(\mathscr{P}^{t}\right)$ is quasi-isomorphic to $G^{t}[-1]$ by Proposition 2.1.1. Since $\mathscr{P}^{t}$ is also $F$-nilpotent, the inclusion $Z\left(\mathscr{P}^{t}\right) \rightarrow Z^{t}$ is a quasi-isomorphism by [13] Corollary 82. This gives the exact upper row of (3.8). The lower row arises from the short exact sequence

$$
0 \rightarrow Q_{\mathcal{N}} \stackrel{F_{1} \text {-incl }}{\longrightarrow} P_{\mathcal{N}} \rightarrow G \rightarrow 0,
$$

which exists because $\mathscr{P}$ is $V$-nilpotent. Here $\underline{\operatorname{Hom}}\left(G, \widehat{\mathbb{G}}_{m}\right)$ vanishes because $\mathscr{P}$ is $F$-nilpotent, thus $G$ unipotent; see Corollary 2.4. 
Finally, let us define $\Delta$ to be the image of $\operatorname{id}_{G^{\vee}}$ under the first row of the following commutative diagram, whose horizontal arrows $\beta$ are given by adjunction ${ }^{(1)}$. The rest of the diagram is used to determine the composition $\Delta \circ \Psi \circ \pi$ and show that the right hand square of (3.8) commutes.

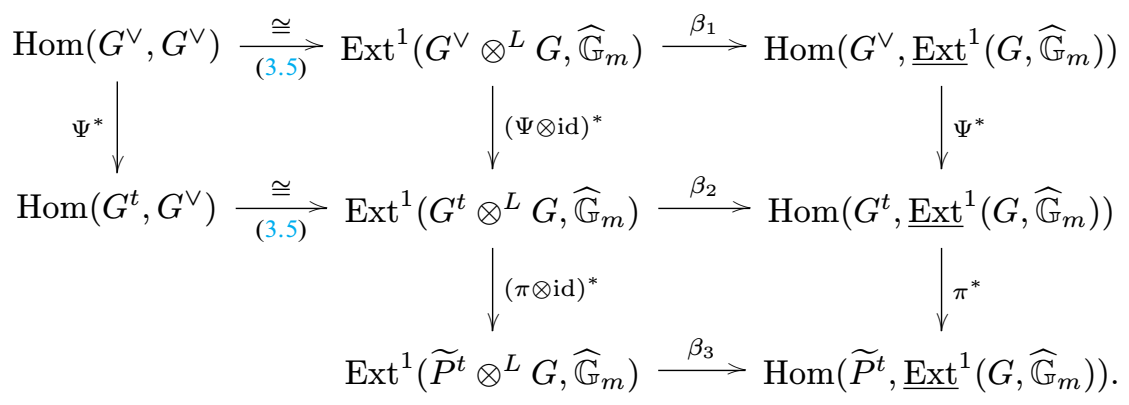

By the definition of $\Psi$, the image of $\operatorname{id}_{G \vee}$ in the middle is equal to $\psi(\alpha)$, where $\alpha$ is the natural bilinear form $\mathscr{P}^{t} \times \mathscr{P} \rightarrow \mathscr{G}_{m}$.

Because of the quasi-isomorphisms $Z \rightarrow Z(\mathscr{P})$ and $Z\left(\mathscr{P}^{t}\right) \rightarrow Z^{t}$, in the construction of $\psi(\alpha)$ we can start with the obvious pairing $\gamma: Z^{t} \otimes Z \rightarrow Z\left(\widehat{\mathbb{G}}_{m}\right) \simeq \widehat{\mathbb{G}}_{m}[-1]$, defined by the second choice of $\gamma_{1}$ on page 252 . Since the double tensor product $\otimes^{L} \mathbb{Q}_{p} / \mathbb{Z}_{p}$ results in a shift by two, $\psi(\alpha)$ gets identified with the composition

$$
G^{t} \otimes^{L} G \stackrel{\simeq}{\longleftarrow} Z^{t}[1] \otimes^{L} Z[1] \stackrel{\text { can }}{\longrightarrow} Z^{t}[1] \otimes Z[1] \stackrel{\gamma[2]}{\longrightarrow} \widehat{\mathbb{G}}_{m}[1] .
$$

Using that $\pi$ is induced by the obvious homomorphism $\widetilde{P}^{t} \rightarrow Z^{t}[1]$, it follows that the composition $\Delta \circ \Psi \circ \pi=\beta_{3}\left((\pi \otimes \mathrm{id})^{*}(\psi(\alpha))\right)$ is equal to the upper line of the following diagram, where $\gamma^{\prime}$ is induced by the pairing $\gamma[2]$, the arrow $\sigma$ is induced by the quasi-isomorphism $Z[1] \rightarrow G$, and $\tau$ is the obvious homomorphism $Z \rightarrow Q_{\mathcal{N}}$.

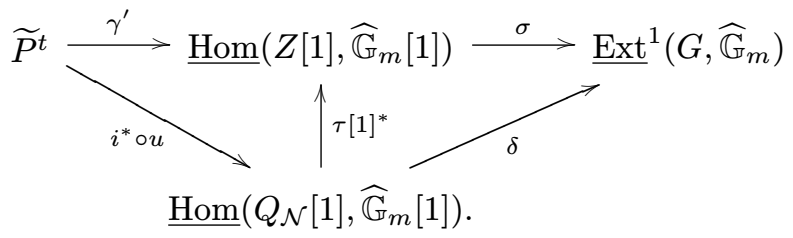

Since both triangles commute, we obtain $\Delta \circ \Psi \circ \pi=\delta \circ i^{*} \circ u$ as desired.

\section{Change of the base ring}

Let $f: R \rightarrow R^{\prime}$ be a local homomorphism of local rings which are admissible in the sense of Definition 1.1. It is no surprise that all preceding constructions are compatible with base change by $f$, i.e. for a Dieudonné display $\mathscr{P}$ over $R$ we have a natural isomorphism

$$
u: \operatorname{BT}\left(\mathscr{P}_{R^{\prime}}\right) \cong \operatorname{BT}(\mathscr{P})_{R^{\prime}},
$$

(1) A simpler definition of $\Delta$, equivalent to the above according to SGA 7, VIII, Proposition 2.3.11 and its correction [1] p. 253, is the following: The restriction of $\Delta$ to $G^{\vee}\left[p^{r}\right]=G\left[p^{r}\right]^{\vee}$ is the connecting homomorphism of the Ext-sequence associated to $0 \rightarrow G\left[p^{n}\right] \rightarrow G \rightarrow G \rightarrow 0$. As $G$ is unipotent in our case, it follows that $\Delta$ is an isomorphism by [7] Theorem 10.2 or by Proposition A.1.

$4^{\text {e }}$ SÉRIE - TOME $42-2009-\mathrm{N}^{\mathrm{o}} 2$ 
transitive with respect to triples $R \rightarrow R^{\prime} \rightarrow R^{\prime \prime}$, such that the following commutes.

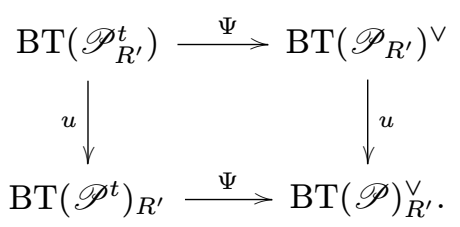

If one uses the original construction of the functor BT in [11], the isomorphism $u$ is quite clear, but for (4.2) we need $u$ in terms of the formulae of Definition 1.6. This is a question about functoriality of the category $\mathcal{C}_{R}$.

Assume first that the residue extension of $R \rightarrow R^{\prime}$ is algebraic. Then every $S \in \mathcal{C}_{R^{\prime}}$ lies in $\mathcal{C}_{R}$ too, and coverings of $S$ in both categories are the same. Hence we have an exact restriction functor $\widetilde{\mathcal{C}_{R}} \rightarrow \widetilde{\mathcal{C}_{R^{\prime}}}$ and (4.1) is evident. By construction of $\psi$, for Dieudonné displays $\mathscr{P}$ and $\mathscr{P}^{\prime}$ over $R$, the following diagram commutes, which gives (4.2).

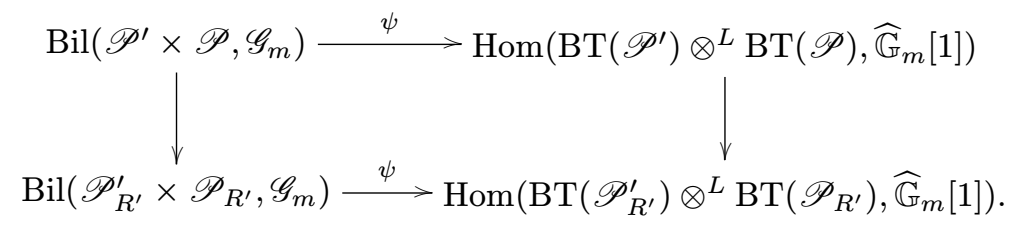

In general we have to modify $\mathcal{C}_{R}$ in order to apply the same reasoning. Let $\mathcal{E}_{R}$ be the category of all $R$-algebras $S$ such that the nilradical $\mathcal{N}_{S}$ is nilpotent, $\mathcal{N}_{S}$ contains $\mathfrak{m} S$, and $S_{\text {red }}=S / \mathcal{N}_{S}$ is perfect. Let $\widetilde{\mathcal{E}}_{R}$ be the category of abelian sheaves on $\mathcal{E}_{R}^{\text {op }}$ for the topology where a covering is a faithfully flat homomorphism $S \rightarrow S^{\prime}$ such that $S_{\text {red }} \rightarrow S_{\text {red }}^{\prime}$ is indétale. The last condition is automatic when $S$ and $S^{\prime}$ lie in $\mathcal{C}_{R}$; conversely for $S \in \mathcal{C}_{R}$ and a covering $S \rightarrow S^{\prime}$ in $\mathcal{E}_{R}$ we necessarily have $S^{\prime} \in \mathcal{C}_{R}$. It follows that coverings of $S \in \mathcal{C}_{R}$ are the same in $\mathcal{C}_{R}$ or in $\mathcal{E}_{R}$, whence an exact restriction functor $\widetilde{\mathcal{E}}_{R} \rightarrow \widetilde{\mathcal{C}}_{R}$.

Now it suffices to note that in all constructions we could use $\mathcal{E}_{R}$ in place of $\mathcal{C}_{R}$. Then (4.1) and (4.2) follow as before since every $S \in \mathcal{E}_{R^{\prime}}$ lies in $\mathcal{E}_{R}$ with the same coverings in both categories. The only point that might need verification is the fact that $p$-divisible groups over $R$ form an exact subcategory of $\widetilde{\mathcal{E}}_{R}$. This follows from the remarks preceding Corollary 2.5 or from:

Lemma 4.1. - If $H$ is a finite flat group scheme over $R$ and $\operatorname{Spec} T \rightarrow \operatorname{Spec} S$ is an $H$-torsor with $S \in \mathcal{E}_{R}$ then $S \rightarrow T$ is a covering in $\mathcal{E}_{R}$.

Proof. - We may assume that $H$ is étale or $H_{k}$ is infinitesimal since any $H$ is an extension of such groups. The étale case is clear. If $H_{k}$ is infinitesimal then the torsor $\operatorname{Spec} T$ is trivial over $S_{\text {red }}$ as $S_{\text {red }}$ is perfect, so $T_{\text {red }} \cong S_{\text {red }}$. 


\section{Appendix}

\section{Infinitesimal extensions of $p$-divisible groups}

For a lack of reference let us mention the following, probably well-known, generalisation of the deformational duality theorem in [7]. Suppose $G, H$ are $p$-divisible groups on an arbitrary scheme $S$ and $S_{\circ}$ is a closed subscheme of $S$. Let $\operatorname{Hom}\left(T_{p} G, H\right)=\underset{\lim }{\longrightarrow} \operatorname{Hom}\left(G\left[p^{n}\right], H\right)$ with transition maps induced by $p: G\left[p^{n+1}\right] \rightarrow G\left[p^{n}\right]$. We have a homomorphism

$$
\delta: \operatorname{Hom}_{S / S_{\circ}}\left(T_{p} G, H\right) \rightarrow \operatorname{Ext}_{S / S_{\circ}}^{1}(G, H)
$$

induced by the exact sequences $0 \rightarrow G\left[p^{n}\right] \rightarrow G \stackrel{p^{n}}{\longrightarrow} G \rightarrow 0$, where $\operatorname{Hom}_{S / S_{\circ}}$ denotes homomorphisms on $S$ which are trivial on $S_{\circ}$ and $\operatorname{Ext}_{S / S_{\circ}}^{1}$ denotes isomorphism classes of extensions on $S$ equipped with a trivialisation on $S_{\text {。 }}$.

Proposition A.1. - If the quasicoherent ideal $I \subseteq \mathcal{O}_{S}$ defining $S_{\circ}$ is nilpotent and annihilated by a power of $p$, then $\delta$ is bijective.

For $H=\mu_{p^{\infty}}$ this results in an isomorphism $G^{\vee}\left(S / S_{\circ}\right) \cong \operatorname{Ext}_{S / S_{\circ}}^{1}\left(G, \mu_{p^{\infty}}\right)$, which is [7] Theorem 10.2, but the isomorphism given there is the negative of $\delta$ by Lemma A.2 below.

Proof of Proposition A.1. - Assume that $p^{r} I=0$ and $I^{n}=0$ and let $m=n r$. The inverse of $\delta$ can be constructed as follows. Assume that $e=[H \rightarrow E \rightarrow G]$ is an extension on $S$ trivialised on $S_{\circ}$, i.e. provided with a section $s_{\circ}: G_{\circ} \rightarrow E_{\circ}$. Then $p^{m} s_{\circ}$ lifts to a unique homomorphism $t: G \rightarrow E$, giving the following morphism of exact sequences.

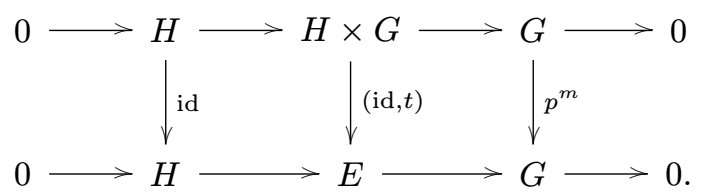

The kernel of (id, $t$ ) is the graph of a homomorphism $f: G\left[p^{m}\right] \rightarrow H$ that is trivial on $S_{\circ}$, and $e \mapsto-f$ is the inverse of $\delta$.

Lemma A.2. - The natural diagram

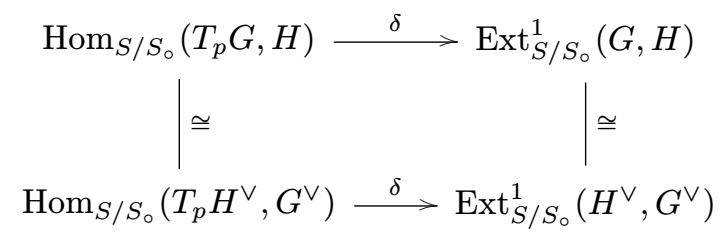

whose vertical isomorphisms are given by Cartier duality is anti-commutative.

Proof. - Let $f \in \operatorname{Hom}_{S / S_{\circ}}\left(T_{p} G, H\right)$ be given and let $0 \rightarrow H \rightarrow E \rightarrow G \rightarrow 0$ be its image under $\delta$. For every sufficiently large $n$ so that $f$ is represented by a homomorphism $f_{n}: G\left[p^{n}\right] \rightarrow H\left[p^{n}\right]$, the truncated extension $E\left[p^{n}\right]$ is naturally isomorphic to the middle cohomology of the following complex, denoted by $K\left(f_{n}\right)$

$$
G\left[p^{n}\right] \stackrel{\left(\mathrm{id},-f_{n}\right)}{\longrightarrow} G\left[p^{n}\right] \oplus H\left[p^{n}\right] \stackrel{\left(f_{n}, \mathrm{id}\right)}{\longrightarrow} H\left[p^{n}\right] .
$$

Since $K\left(f_{n}\right)^{\vee} \cong K\left(-f_{n}^{\vee}\right)$ the assertion follows. 


\title{
REFERENCES
}

[1] P. Berthelot, L. Breen, W. Messing, Théorie de Dieudonné cristalline II, Lecture Notes in Math. 930, Springer, 1982.

[2] C. Breuil, Groupes p-divisibles, groupes finis et modules filtrés, Ann. of Math. 152 (2000), 489-549.

[3] P. CArtier, Groupes formels associés aux anneaux de Witt généralisés, C. R. Acad. Sci. 265 (1976), 50-52.

[4] X. Caruso, Dualité de Cartier et modules de Breuil, preprint arXiv:math/0511423.

[5] E. LAU, Displays and formal p-divisible groups, Invent. Math. 171 (2008), 617-628.

[6] H. Matsumura, Commutative ring theory, Cambridge Studies in Advanced Mathematics 8, Cambridge University Press, 1986.

[7] B. Mazur, W. Messing, Universal extensions and one dimensional crystalline cohomology, Lecture Notes in Math. 370, Springer, 1974.

[8] W. Messing, The crystals associated to Barsotti-Tate groups: with applications to abelian schemes, Lecture Notes in Math. 264, Springer, 1972.

[9] D. Mumford, Bi-extensions of formal groups, in Algebraic Geometry (Internat. Colloq., Tata Inst. Fund. Res., Bombay, 1968), Oxford Univ. Press, 1969, 307-322.

[10] P. Norman, An algorithm for computing local moduli of abelian varieties, Ann. Math. 101 (1975), 499-509.

[11] T. ZINK, A Dieudonné theory for $p$-divisible groups, in Class field theory - its centenary and prospect (Tokyo, 1998), Adv. Stud. Pure Math. 30, Math. Soc. Japan, 2001, 139160.

[12] T. ZINK, Windows for displays of $p$-divisible groups, in Moduli of abelian varieties (Texel Island, 1999), Progr. Math. 195, Birkhäuser, 2001, 491-518.

[13] T. ZINK, The display of a formal p-divisible group, Astérisque 278 (2002), 127-248.

(Manuscrit reçu le 26 novembre 2007;

accepté, après révision, le 13 novembre 2008.)

\author{
Eike LAU \\ Fakultät für Mathematik \\ Universität Bielefeld \\ Postfach 100131 \\ D-33501 Bielefeld, Germany \\ E-mail: lau@math.uni-bielefeld.de
}

